November 20, 2018

\title{
Supersymmetry and Dark Matter
}

\author{
R. Arnowitt And B. Dutta \\ Center For Theoretical Physics, Department of Physics, \\ Texas A\&M University, College Station TX 77843-4242
}

We examine supergravity models with grand unification at $M_{G}$ possessing $\mathrm{R}$ parity invariance. Current data has begun to significantly constrain the parameter space. Thus for mSUGRA, accelerator data places a lower bound on $m_{1 / 2}$ of $m_{1 / 2} \gtrsim 300 \mathrm{GeV}$ while astronomical data on the amount of relic dark matter narrowly determines $m_{0}$ in terms of $m_{1 / 2}$ (for fixed value of $\tan \beta$ and $A_{0}$ ) due to co-annihilation effects. Additional new data could fix the parameters further. Thus the parameter space is sensitive to the muon magnetic moment anomaly, $\delta a_{\mu}$, and if $\delta a_{\mu}$ lies $1 \sigma$ above its current central value, it would exclude mSUGRA, while if it lies $1 \sigma$ below (but is still positive) it pushes the SUSY spectrum into the TeV domain. The $B_{s} \rightarrow \mu^{+} \mu^{-}$decay is seen to be accessible to the Tevatron RunII with branching ratio sensitivity of $\operatorname{Br}\left[B_{s} \rightarrow \mu^{+} \mu^{-}\right]>6.5 \times 10^{-9}$ with $15 \mathrm{fb}^{-1} /$ detector, and a value of $7(14) \times 10^{-8}$ obtainable with $2 \mathrm{fb}^{-1}$ would be sufficient to exclude mSUGRA for $\tan \beta<50(55)$. Measurements of $B_{s} \rightarrow \mu^{+} \mu^{-}$can cover the full mSUGRA parameter space for $\tan \beta>40$ if $\delta a_{\mu}>11 \times 10^{-10}$, and combined measurements of $B_{s} \rightarrow \mu^{+} \mu^{-}, a_{\mu}$ and $m_{h}$ (or alternately the gluino mass) would effectively determine the mSUGRA parameters for $\mu>0$. Detector cross sections are then within the range of planned future dark matter experiments. Non-universal models are also discussed, and it is seen that detector cross sections there can be much larger, and can be in the DAMA data region.

PRESENTED AT

DARK 2002

Cape Town, South Africa

4-9 February, 2002 


\section{Introduction}

It is generally expected that the Standard Model will break down at energies above LEP, and signals of new physics will occur for energies $\gtrsim 100 \mathrm{GeV}-1 \mathrm{TeV}$. The nature of this new physics is one of the crucial question of particle physics. Simultaneously, astronomical data has determined with good accuracy the amount of dark matter in the universe, though the nature of that dark matter remains one of the crucial questions of astronomy. Supersymmetric theories with $\mathrm{R}$ parity invariance offer an explanation to both puzzles as well as a window on the cosmology of the very early universe at times $t \approx 10^{-7} \mathrm{sec}$.

Unfortunately, supersymmetric models depend upon a large number of parameters, and even the simplest model, mSUGRA, depends on four parameters and one sign. But fortunately, supersymmetry applies to a wide number of phenomena, and it is now becoming possible to significantly restrict the parameter space. The general MSSM, with over 100 free parameters (63 real parameters) is not very predictive. We consider here therefore, models, based on supergravity grand unification at $M_{G} \simeq 2 \times 10^{16} \mathrm{GeV}$ (which have both theoretical and experimental motivation). We examine first the current status of the simplest model, mSUGRA [1, 2], and what might be obtained from future measurements of the muon magnetic moment, $g_{\mu}-2$, the Higgs mass, and the B decay $B_{s} \rightarrow \mu^{-} \mu^{+}$. We will then look at non-universal models with non-universality in the Higgs or third generation of squarks and sleptons. For all these cases, the lightest neutralino, $\tilde{\chi}_{1}^{0}$, is the dark matter candidate, and this will also strongly constrain the SUGRA parameter space.

\section{2 mSUGRA Model}

We briefly review the mSUGRA model which depends on four parameters and one sign, and thus is the most predictive of the SUSY models. We take these parameters to be the following: $m_{0}$ (the universal scalar soft breaking mass at $M_{G}$ ); $m_{1 / 2}$ (the universal gaugino mass at $M_{G}$ ); $A_{0}$ (the universal cubic soft breaking mass at $M_{G}$ ); and $\tan \beta=<H_{2}>$ $\left./<H_{1}\right\rangle$ (the ratio of the two Higgs VEVs at the electroweak scale). The sign of the Higgs mixing parameter $\mu$ (appearing in the superpotential as $\mu H_{1} H_{2}$ ) is the remaining parameter. (We note at the electroweak scale that the $\tilde{\chi}_{1}^{0}$ and lightest chargino, $\tilde{\chi}_{1}^{ \pm}$, masses are related to $m_{1 / 2}$ by $m_{\tilde{\chi}_{1}^{0}} \cong 0.4 m_{1 / 2}$ and $m_{\tilde{\chi}_{1}^{ \pm}} \cong 0.8 m_{1 / 2}$.) We examine this model with the following parameter ranges: $m_{0} \leq 1 \mathrm{TeV} ; m_{1} / 2 \leq 1 \mathrm{TeV} ; 2 \leq \tan \beta \leq 55$; and $A_{0} \leq 4 m_{1 / 2}$. The above bound on $m_{1 / 2}$ corresponds to a gluino mass range of $m_{\tilde{g}} \leq 2.5$ $\mathrm{TeV}$, which is also the upper mass reach for the LHC.

In the early universe, the neutralino can annihilate via s-channel $Z$, and $h, H$, and $A$ neutral Higgs bosons ( $h$ is the light Higgs, and $H(A)$ are the heavy CP even (odd) Higgs), and also through t-channel sfermion diagrams. However, if a second particle becomes nearly degenerate with the $\tilde{\chi}_{1}^{0}$, one must include it in the early universe annihilation processes. This leads to the co-annihilation phenomena. In SUGRA models with gaugino grand unification, this accidental near degeneracy occurs naturally for the light stau, $\tilde{\tau}_{1}$. 
One can see this analytically for low and intermediate $\tan \beta$, where the renormalization group equations (RGE) can be solved analytically. One finds for $\tilde{e}_{R}$, the right selectron and the $\tilde{\chi}_{1}^{0}$ at the electroweak scale the results:

$$
\begin{aligned}
& m_{\tilde{e}_{R}}=m_{0}^{2}+0.15 m_{1 / 2}^{2}-\sin ^{2} \theta_{W} M_{W}^{2} \cos 2 \beta \\
& m_{\tilde{\chi}_{1}^{0}}=0.16 m_{1 / 2}^{2}
\end{aligned}
$$

where the last term of $\mathrm{Eq}(1)$ is approximately $(37 \mathrm{GeV})^{2}$. Thus for $m_{0}=0, \tilde{e}_{R}$ becomes degenrerate with $\tilde{\chi}_{1}^{0}$ at $m_{1 / 2} \cong 370 \mathrm{GeV}$, and co-annihilation thus begins at $m_{1 / 2} \cong(350 \mathrm{GeV}$ - 400GeV). As $m_{1 / 2}$ increases, $m_{0}$ must be raised in lock step (to keep $m_{\tilde{e}_{R}}>m_{\tilde{\chi}_{1}^{0}}$ ). More precisely, it is the $\tilde{\tau}_{1}$ which is the lightest slepton and this particle dominates the co-annihilation phenomena. In general, co-annihilation implies that one ends up with relatively narrow allowed corridors in the $m_{0}-m_{1 / 2}$ plane with $m_{0}$ closely correlated with $m_{1 / 2}$, increasing as $m_{1 / 2}$ increases.

Dark matter detection of Milky Way neutralinos incident on the Earth depends upon the neutralino - proton cross section. For detectors with nuclear targets containing heavy nuclei, this is dominated by the spin independent cross section. The basic quark diagrams involve s-channel squarks, and $t$-channel $h$ and $H$ diagrams. Thus $\sigma_{\tilde{\chi}_{1}^{0}-p}$ decreases with increasing $m_{1 / 2}$ and $m_{0}$ (which as we have seen above increase together), and also increases with $\tan \beta$ (due to the Higgs couplings to the $d$ quark). Thus the maximum cross section will occur at high $\tan \beta$, and low $m_{1 / 2}, m_{0}$.

In order to carry out calculations in SUGRA models accurately, it is necessary to take into account a number of corrections, and we list the important ones here:

We use two loop gauge and one loop Yukawa RGE from $M_{G}$ to the electroweak scale (which we take as $\left(\tilde{t}_{1} \tilde{t}_{2}\right)^{1 / 2}$ ), and QCD RGE below for the light quark contributions. Two loop and pole mass corrections are included in calculation of $m_{h}$. One loop corrections to $m_{b}$ and $m_{\tau}$ [3, 4] are included, which are important for large $\tan \beta$. Large tan $\beta$ NLO SUSY corrections to $b \rightarrow s \gamma$ [5, 6] are included. All stau-neutralino co-annihilation channels are included in the relic density calculation with analysis valid for the large $\tan \beta$ regime [7, 8, 9].

Note that we do not include Yukawa unification or proton decay constraints in the analysis as these depend sensitively on post-GUT physics, about which little is known. In fact in string or M-theory analyses with grand unification, while the unification of the coupling constants occur as in SUGRA models, the Yukawa unification or proton decay constraints do not generally hold [10].

\section{Current Experimental Constraints}

In order to see what the currently allowed parameter space is, one must impose all the present experimental constraints. However, three of these acting together produce significant limitations, and we mention these here: 


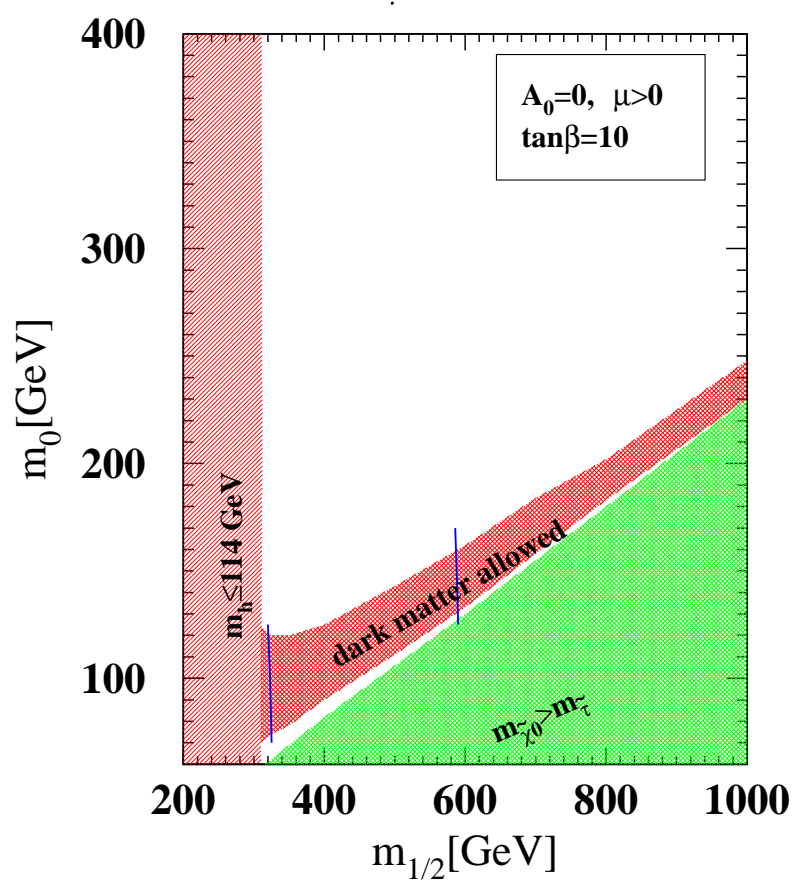

Figure 1: $m_{0}-m_{1 / 2}$ plane showing the allowed parameter region for $\tan \beta=10, A_{0}=0$, $\mu>0$. The short vertical lines give $\sigma_{\tilde{\chi}_{1}^{0}-p}=5 \times 10^{-9} \mathrm{pb}$ (left line) and $1 \times 10^{-9} \mathrm{pb}$ (right line).

(1) Higgs mass. The current LEP bound on the light Higgs is $m_{h}>113.5 \mathrm{GeV}$ 11]. However, the theoretical calculation of $m_{h}$ [12] may have a $2-3 \mathrm{GeV}$ error, and so we will conservatively interpret this bound to mean $m_{h}$ (theory) $>111 \mathrm{GeV}$.

(2) $b \rightarrow s \gamma$ decay. There is some model dependence in extracting the $b \rightarrow s \gamma$ branching ratio from the CLEO data, and so we will take a relatively broad range around the current CLEO central value [13]:

$$
1.8 \times 10^{-4}<\operatorname{Br}\left(B \rightarrow X_{s} \gamma\right)<4.5 \times 10^{-4}
$$

(3) $\tilde{\chi}_{1}^{0}$ relic density. The relic density is measured in terms of $\Omega=\rho / \rho_{c}$ where $\rho$ is the mass density, $\rho_{c}=3 H^{2} / 8 \pi G_{N}$ and $H=(100 \mathrm{~km} / \mathrm{sMpc}) h$ is the Hubble constant. Analyses of the cosmic microwave background now gives a fairly accurate measurement of the amount of CDM, i. e. $\Omega_{\mathrm{CDM}} h^{2}=0.139 \pm 0.026$ [14]. We take a $2 \sigma$ range around the central value:

$$
0.07<\Omega_{\tilde{\chi}_{1}^{0}} h^{2}<0.21
$$

These three constraints now combine to greatly restrict the mSUGRA parameter space. Thus the $m_{h}$ bound for low $\tan \beta$ and the $b \rightarrow s \gamma$ constraint for higher $\tan \beta$ produce a 


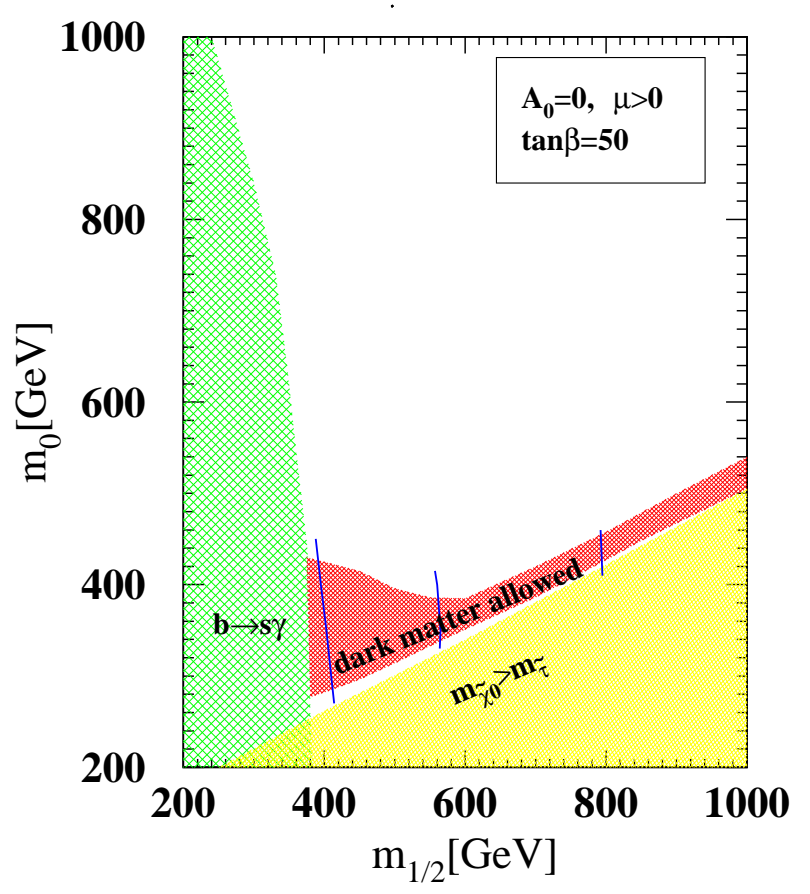

Figure 2: $m_{0}-m_{1 / 2}$ plane showing the allowed parameter region for $\tan \beta=50, A_{0}=0$, $\mu>0$.The short vertical lines give (from left to right) $\sigma_{\tilde{\chi}_{1}^{0}-p}=5 \times 10^{-8} \mathrm{pb}, 1 \times 10^{-8} \mathrm{pb}$, $2 \times 10^{-9} \mathrm{pb}$.

lower bound on $m_{1 / 2}$ over the entire parameter space of

$$
m_{1 / 2} \gtrsim(300-400) \mathrm{GeV}
$$

and consequently $m_{\tilde{\chi}_{1}^{0}} \gtrsim(120-160) \mathrm{GeV}$. This means that most of the parameter space is in the $\tilde{\tau}_{1}-\tilde{\chi}_{1}^{0}$ co-annihilation domain in the relic density calculation, and thus to satisfy the relic density bound, $m_{0}$ is approximately determined by $m_{1 / 2}$ (for fixed $\tan \beta, A_{0}$ ). This implies that as $m_{1 / 2}$ increases, so does $m_{0}$, and so generally on has that $\sigma_{\tilde{\chi}_{1}^{0}-p}$ is a deceasing function of $m_{1 / 2}$.

We consider first $\mu>0$. Figs. 1 and 2 exhibit the effects discussed above. Thus in Fig. 1 for $\tan \beta=10, A_{0}=0$, one sees that the Higgs mass bound requires $m_{1 / 2} \gtrsim 300$ $\mathrm{GeV}$, and one sees the narrow $m_{0}$ band allowed by the co-annihilation effects. The short vertical lines show the expected dark matter detection cross sections of $\sigma_{\tilde{\chi}_{1}^{0}-p}=5^{-9} \mathrm{pb}$ (left) and $1 \times 10^{-9} \mathrm{pb}$ (right). Thus $m_{0}$ is determined by $m_{1 / 2}$ to within $\sim 40 \mathrm{GeV}$. Fig. 2 shows the corresponding situation for $\tan \beta=50$. Here the $b \rightarrow s \gamma$ produces the low energy cut off of $m_{1 / 2} \gtrsim 350 \mathrm{GeV}$. There is also a bulge in the dark matter allowed domain at low $m_{1 / 2}$ where co-annihilation has not yet set in. The cross sections are (left to right) 
$5 \times 10^{-8} \mathrm{pb}, 1 \times 10^{-8} \mathrm{pb}$ and $2 \times 10^{-9} \mathrm{pb}$. These cross sections are within range of future planned detector experiments.

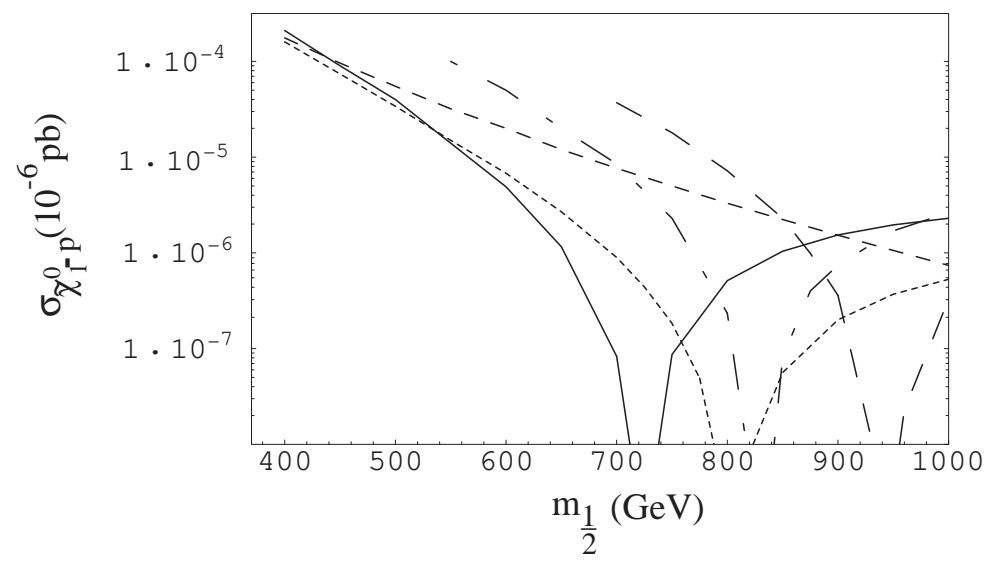

Figure 3: Minimum values of $\sigma_{\tilde{\chi}_{1}^{0}-p}$ as a function of $m_{1 / 2}$ for $\mu<0, \tan \beta=6$ (dashed), 8 (dotted), 10 (solid) and 25 (large dash)[7].

For $\mu<0$ an accidental cancellation in $\sigma_{\tilde{\chi}_{1}^{0}-p}$ occurs over a wide range of $\tan \beta$ [15, [7] giving large regions where $\sigma_{\tilde{\chi}_{1}^{0}-p}<10^{-10} \mathrm{pb}$ and hence probably inaccessible to future detectors. This is exhibited in Fig. 3 [7], where the minimum cross sections are plotted as one scans the allowed parameter space, for $\tan \beta=6$ (dashed), 8 (dotted), 10 (solid), 25 (large dash). In this case the spin independent cross section can fall below the very small spin dependent cross section where such cancellations do not occur 16.

There exist now two other experiments that might restrict the parameter space even more: The BNL E821 [17] $g_{\mu}-2$ experiment measuring the muon magnetic moment, and the decay $B_{s} \rightarrow \mu^{+} \mu^{-}$which may be observable at the Tevatron RunIIB (or possibly in B-factories), and we turn to consider these next.

\section{Muon magnetic Moment Anomaly}

The BNL E821 experiment has measured the muon magnetic moment with exceedingly high accuracy. When compared with the calculations of $a_{\mu}$ expected from the Standard Model (with corrected sign of the hadronic scattering of light by light contribution [18]) there remains a small discrepancy:

$$
\delta a_{\mu}=27(16) \times 10^{-10}
$$

which is a $1.6 \sigma$ effect. While this is not enough to presume that a real effect has been discovered, it is still interesting to examine the effects such an anomaly would have for two reasons: first the errors will shortly be significantly reduced, and second SUGRA models imply the existence of such an anomaly of just this size. 


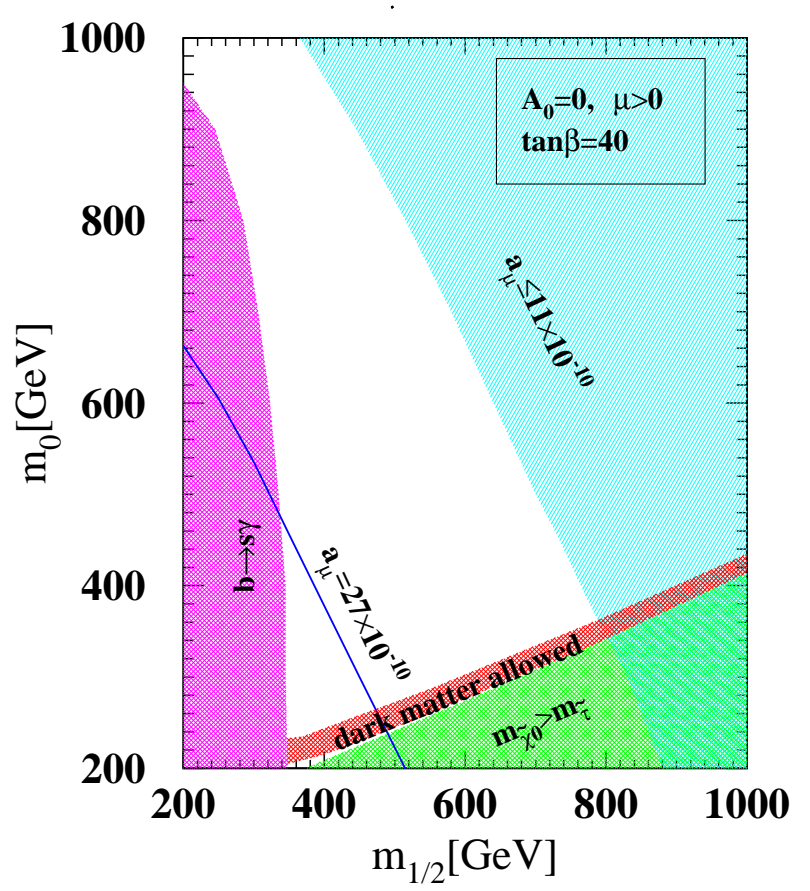

Figure 4: Allowed parameter space in the $m_{0}-m_{1 / 2}$ plane for $\tan \beta=40, A_{0}=0$, $\mu>0$. The upper (blue) region would be eliminated if $a_{\mu}<11 \times 10^{-10}$, and the line for $a_{\mu}=27 \times 10^{-10}$ is shown.

Much of the uncertainty in the calculation of $a_{\mu}$ comes from the hadronic part, $a_{\mu}(\mathrm{had})$. However, it is possible to express this by a dispersion relation in terms of integrals over the experimental cross section $\sigma_{e^{+} e^{-}} \rightarrow$ had. Recent experiments from from CMD-2, VEPP$2 \mathrm{M}$ and Beijing [19, 20, 21] have re-measured these cross sections with greatly improved accuracy. Further, the BNL E821 experiment has about six times more data which they are currently analyzing which should greatly reduce the statistical part of the error. Thus one may expect the error in Eq.(5) to be reduced by a factor of two or more in the near future. If the central value of the anomaly were to remain unchanged, the effect would become more statistically significant.

From the theoretical side, it has been known for some time that mSUGRA predicts an important contribution to $a_{\mu}$ [22, 23]. This is illustrated in Fig. 4 which shows the allowed regions in the $m_{0}-m_{1 / 2}$ plane and assumes that the entire $a_{\mu}$ anomaly is due to SUSY. The upper right (blue) region corresponds to $a_{\mu}$ less than $1 \sigma$ below the current central value, while the diagonal line corresponds to the central value, which falls at the lower edge of the allowed part of the parameter space. The current central value thus corresponds to a relatively low mass SUSY spectra, easily accessible to the LHC. In fact, too big a value of delta $a_{\mu}$ (i. e. delta $a_{\mu} \gtrsim 40 \times 10^{-10}$ ) would be sufficient to exclude 


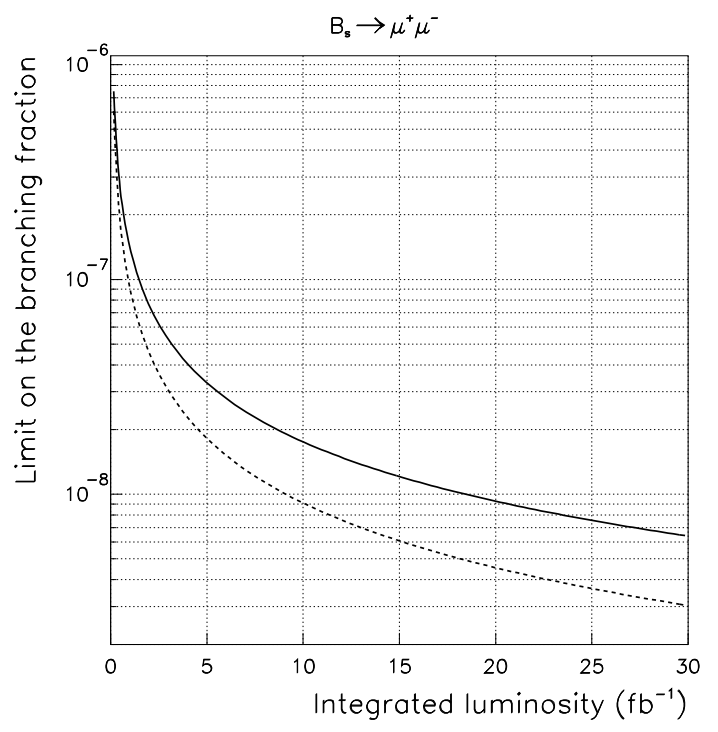

Figure 5: Illustrated 95\% C.L. limits on the branching ratio for $B_{s} \rightarrow \mu^{+} \mu^{-}$at CDF in Run II as a function of integrated luminosity. Solid (Case A) and dashed (Case B) curves are based on different assumptions on the signal selection efficiency and the background rejection power[30].

mSUGRA [24]. On the other hand, an $a_{\mu}$ less than $1 \sigma$ below the current central value would imply a heavy SUSY mass spectrum. Further, if $\delta a_{\mu}$ is positive, then $\mu>0$ [25, 26], and this would eliminate the very low dark matter detection cross sections shown in Fig.3. Thus the final value of $\delta a_{\mu}$ will play an important role in deciphering the SUSY parameter space.

\section{$5 \quad B_{s} \rightarrow \mu^{+} \mu^{-}$}

The $B_{s} \rightarrow \mu^{+} \mu^{-}$decay offers an additional window for investigating the mSUGRA parameter space. This process has been examined within the MSSM framework [27, 28] and more recently using mSUGRA [29]. We consider here predictions for this decay for mSUGRA, but include all the current experimental constraints listed in Sec. 3 (which are necessary to see what predictions occur [30]). The $B_{s} \rightarrow \mu^{+} \mu^{-}$decay is of interest since the Standard Model prediction for the branching ratio is quite small $28\left(\operatorname{Br}\left[B_{s} \rightarrow \mu^{+} \mu^{-}\right]=(3.1 \pm 1.4) \times 10^{-9}\right)$, while the SUSY contribution can become quite large for large $\tan \beta$. This is because the leading diagrams grow as $(\tan \beta)^{3}$ and hence the branching ratio as $(\tan \beta)^{6}$. What further makes this decay interesting is that it is possible to find a set of cuts so that CDF (and probably also DO) may be able to observe it in Run2B (with $15 \mathrm{fb}^{-1}$ data). Fig. 5 shows the CDF limit on the branching fraction as a function of the luminosity [30]. (The solid curve is a conservative estimate, 


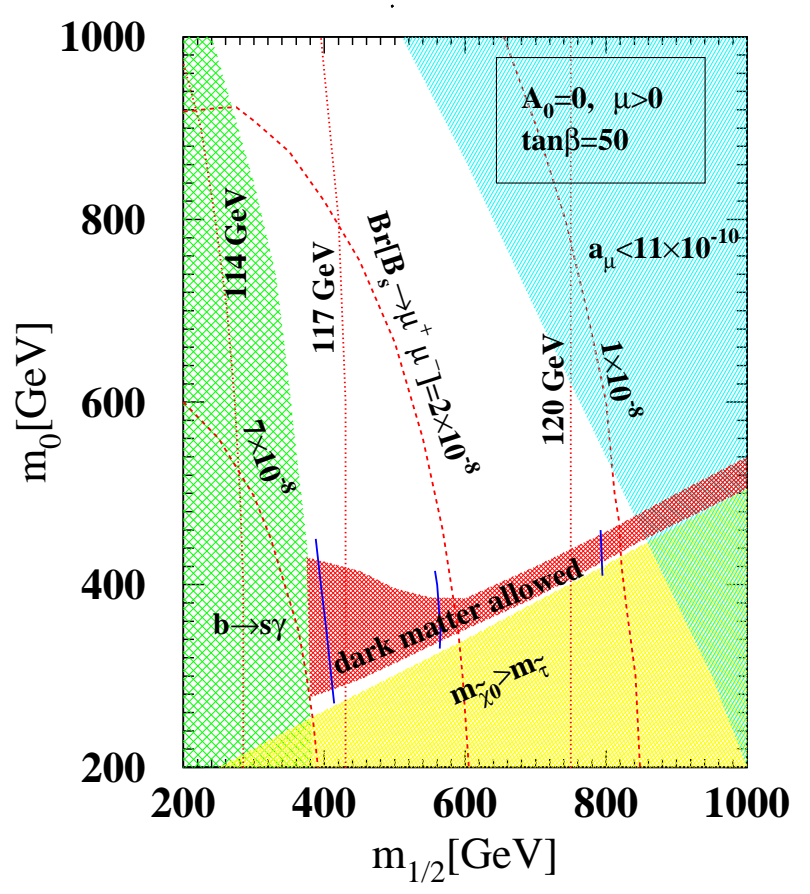

Figure 6: Branching ratio for $B_{s} \rightarrow \mu^{+} \mu^{-}$(three dashed lines from left to right: $7 \times 10^{-8}$, $\left.2 \times 10^{-8}, 1 \times 10^{-8}\right)$ for $\tan \beta=50$ in the $m_{0}-m_{1 / 2}$ plane. Other mSUGRA parameters are fixed to be $A_{0}=0$ and $\mu>0$. The three short solid lines indicate the $\sigma_{\tilde{\chi}_{1}^{0}-p}$ values (from left: $0.05 \times 10^{-6} \mathrm{pb}, 0.004 \times 10^{-6} \mathrm{pb}, 0.002 \times 10^{-6} \mathrm{pb}$ ). The vertical dotted lines label Higgs masses[30].

and the dotted curve a more optimistic possibility.) One sees that CDF will be sensitive to a branching ratio of $\mathrm{Br}>1.2 \times 10^{-8}$ (and the combined CDF and D0 data to $\mathrm{Br}>6.5 \times 10^{-9}$ ). mSUGRA analysis then shows that CDF would be sensitive to this decay for $\tan \beta \gtrsim 30$.

We can now examine the effects of the combination of all data. Figs. 6 and 7 [30] [show the parameter space for $\tan \beta=50$ and $\tan \beta=40$ respectively for $A_{0}=0$ and $\mu>0$. One sees that there is an upper bound on the $\operatorname{Br}\left[B_{s} \rightarrow \mu^{+} \mu^{-}\right]$to be consistent with mSUGRA, and a branching ratio $>7(14) \times 10^{-8}$ would be able to exclude mSUGRA for $\tan \beta<50(55)$. As can be seen from Fig. 5, such a branching ratio could be seen with only $2 \mathrm{fb}^{-1}$. More generally, Fig. 6 shows that the entire parameter space can be covered for $\tan \beta=50, A_{0}=0$ if $a_{\mu}>11 \times 10^{-10}$, and from Fig. 7 also for $\tan \beta=40$ using the combined CDF and D0 data. The effect of varying $A_{0}$ is shown in Fig. 8 where $A_{0}=-2 m_{1 / 2}$, and $\tan \beta=40$. Here again the full parameter space can be covered.

One sees from the above graphs that future measurements should be able to determine the basic parameters of the mSUGRA model. Thus when the lines for $\delta a_{\mu}, \operatorname{Br}\left[B_{s} \rightarrow\right.$ 


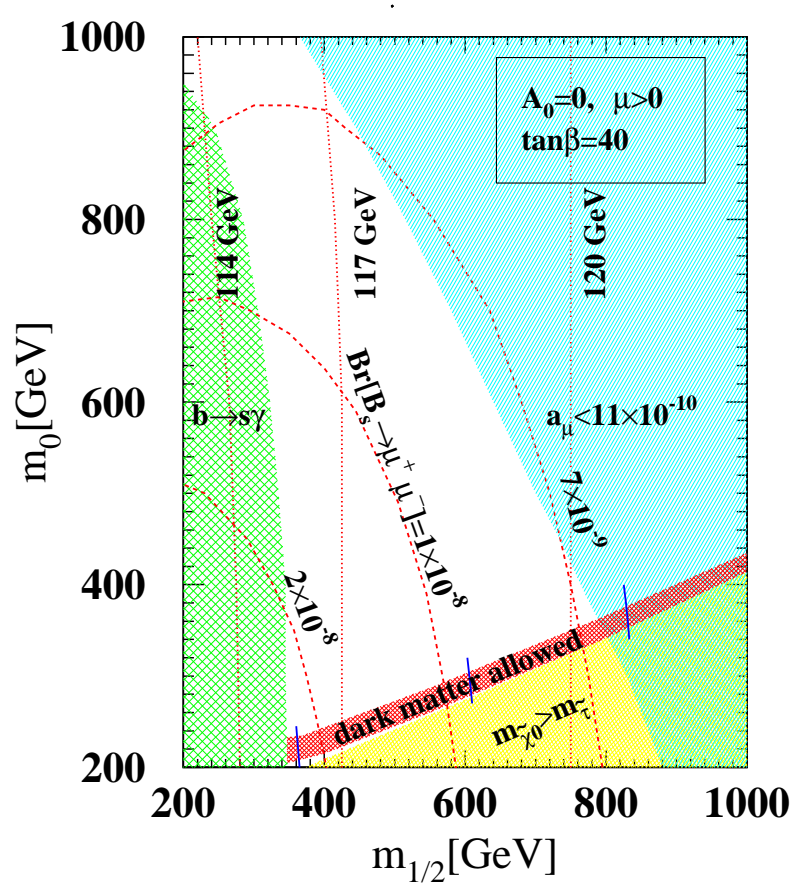

Figure 7: Branching ratio for $B_{s} \rightarrow \mu^{+} \mu^{-}$(three dashed lines from left to right: $1.9 \times 10^{-8}$, $\left.1 \times 10^{-8}, 0.7 \times 10^{-8}\right)$ at $\tan \beta=40$ in the $m_{0}-m_{1 / 2}$ plane. Other mSUGRA parameters are fixed to be $A_{0}=0$ and $\mu>0$. The three short solid lines indicate the $\sigma_{\tilde{\chi}_{1}^{0}-p}$ values (from left: $0.03 \times 10^{-6} \mathrm{pb}, 0.002 \times 10^{-6} \mathrm{pb}, 0.001 \times 10^{-6} \mathrm{pb}$ ). The vertical dotted lines label Higgs masses[30].

$\mu^{+} \mu^{-}$] and $m_{h}$ intersect the allowed dark matter band at a single "point" for a given $A_{0}$, this will determine $m_{0}$ (approximately), $m_{1 / 2}$ and $\tan \beta$. (It may be better to use the gluino mass in place of $m_{h}$ since the parameter space is very sensitive to $m_{h}$.) Predictions of the SUSY spectrum and dark matter detection rates would then follow.

\section{Non-Universal Models}

One can generalize mSUGRA by allowing non-universal soft breaking at $M_{G}$ in the third generation of squarks and sleptons and also in the Higgs sector. If universality of the gaugino masses is maintained, then stau-neutralino co-annihilation will still play an important role. However, new effects can occur, since the non-universality effects the size of the $\mu$ parameter. The $\mu$ parameter governs the Higgsino content of the $\tilde{\chi}_{1}^{0}$ and as $\mu^{2}$ decreases (increases), the Higgsino content increases (decreases). Since $\sigma_{\tilde{\chi}_{1}^{0}-p}$ depends on the interference between the Higgsino and gaugino parts of $\tilde{\chi}_{1}^{0}, \sigma_{\tilde{\chi}_{1}^{0}-p}$ will correspondingly increase 


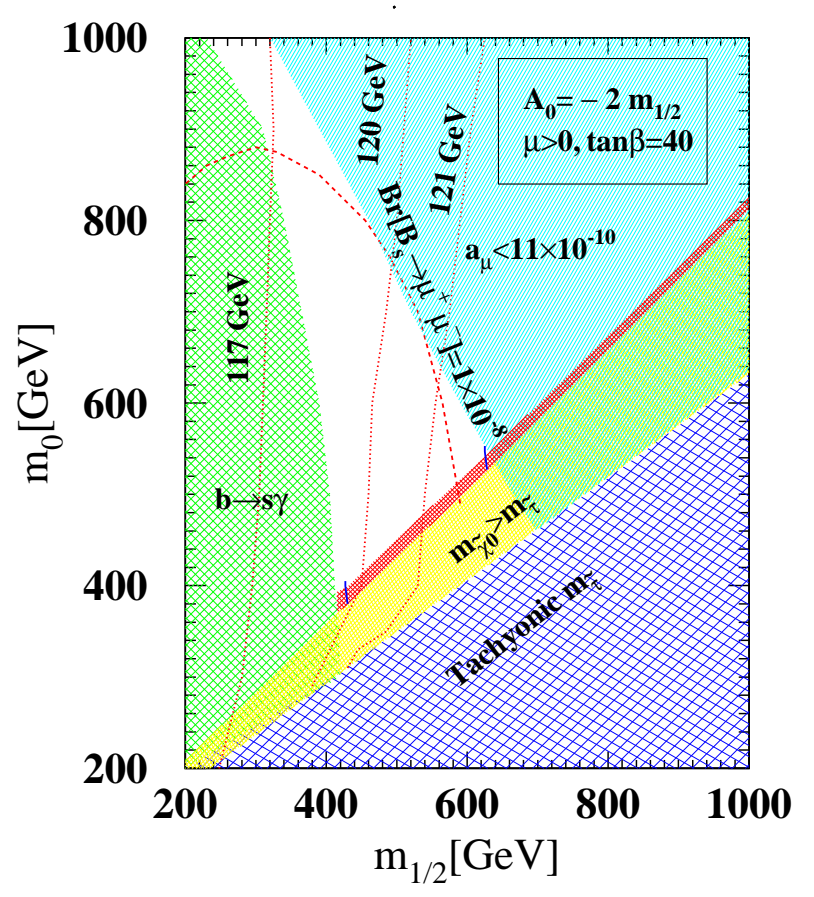

Figure 8: Branching ratio for $B_{s} \rightarrow \mu^{+} \mu^{-}$at $\tan \beta=40$ in the $m_{0}-m_{1 / 2}$ plane for $A_{0}=-2 m_{1 / 2}$ and $\mu>0$. The two short solid lines indicate the $\sigma_{\tilde{\chi}_{1}^{0}-p}$ values (from left: $\left.0.005 \times 10^{-6} \mathrm{pb}, 0.001 \times 10^{-6} \mathrm{pb}\right)$. The vertical dotted lines label Higgs masses[30].

(decrease). A second effect also occurs. As the Higgsino content of the $\tilde{\chi}_{1}^{0}$ increases, then the $\tilde{\chi}_{1}^{0}-\tilde{\chi}_{1}^{0}-Z$ coupling is strengthened, allowing a new annihilation channel to become important (in addition to the $\tilde{\tau}_{1}-\tilde{\chi}_{1}^{0}$ co-annihilation channel). As a simple example we consider the case where at $M_{G}$ one chooses $m_{H_{2}}^{2}=m_{0}^{2}\left(1+\delta_{2}\right)$, and all other soft breaking masses universal. Fig. 9 shows the allowed region in the $m_{0}-m_{1 / 2}$ plane for $\tan \beta=40$, $\delta_{2}=1, A_{0}=0, \mu>0$. One sees the usual narrow stau-neutralino co-annihilation band a relatively low $m_{0}$, but in addition there is a higher $m_{0}$ (and low $m_{1 / 2}$ ) region satisfying all constraints due to the new $Z$-channel annihilation process. Fig. 10 shows $\sigma_{\tilde{\chi}_{1}^{0}-p}$ as a function of $m_{1 / 2}$ for $\tan \beta=40, A_{0}=m_{1 / 2}, \mu>0$. The Z-channel corridor now reaches up to the DAMA data region [31] for low $m_{1 / 2}\left(m_{\tilde{\chi}_{1}^{0}} \simeq 0.4 m_{1 / 2}\right)$, and so if the DAMA results are confirmed, it would point to a non-universality of this type.

\section{Conclusions}

We have considered here SUGRA models with $\mathrm{R}$ parity invariance and grand unification at $M_{G} \simeq 2 \times 10^{16} \mathrm{GeV}$. Current data has begun to constraint these models significantly. 


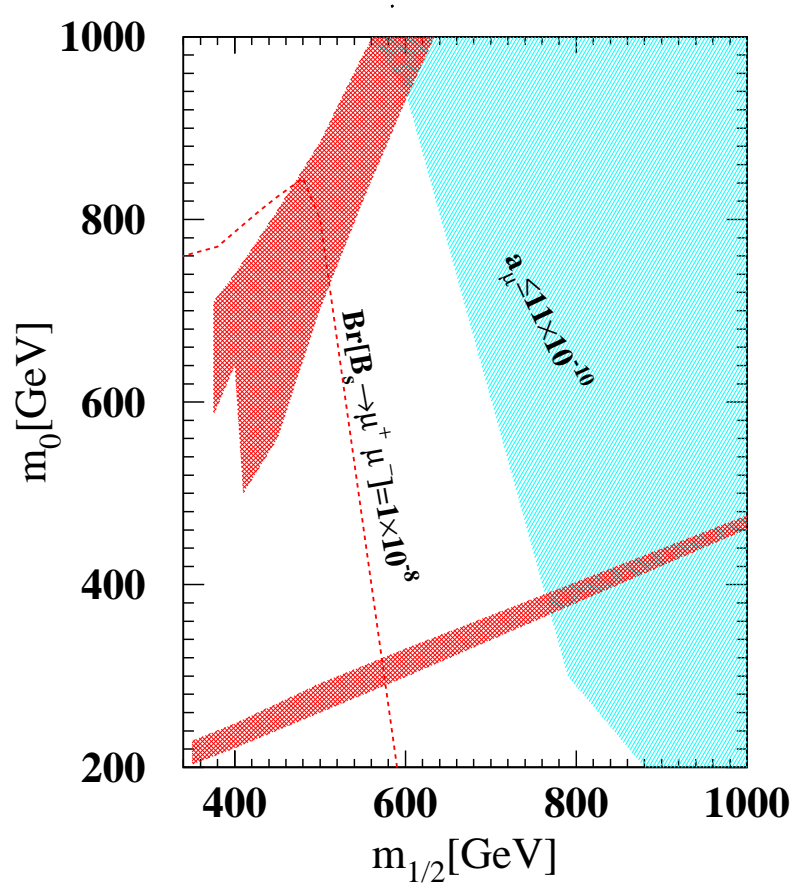

Figure 9: Allowed regions in the $m_{0}-m_{1 / 2}$ plane for $\delta_{2}=1, \tan \beta=40, A_{0}=0, \mu>0$. The lower band is the ususal $\tilde{\tau}_{1}-\tilde{\chi}_{1}^{0}$ co-annihilation channel, and the upper region is the new $Z$ boson s-channel annihilation region.

For mSUGRA (and many non-universal models), accelerator data ( $m_{h}$ and $b \rightarrow s \gamma$ ) place lower bounds on $m_{1 / 2}$ such that $m_{1 / 2} \gtrsim 300 \mathrm{GeV}$ (or $m_{\tilde{\chi}_{1}^{0}} \gtrsim 120 \mathrm{GeV}$ ), while astronomical data on the amount of dark matter narrowly determines $m 0$ in terms of $m_{1 / 2}$ (for each $A_{0}$ and $\tan \beta$ ).

Thus additional new data could begin to fix the parameters of mSUGRA parameters, and so determine the mass spectrum expected at the LHC, dark matter detection rates, etc. In particular, the muon magnetic moment anomaly $a_{\mu}$ and the branching ratio $\operatorname{Br}\left[B_{s} \rightarrow \mu \mu\right]$ could combine with $m_{h}$ or a measurement of $m_{\tilde{g}}$ to fix the parameters completely. A more accurate determination of $a_{\mu}$ should be available shortly, and we have seen that the Tevatron RunII should be sensitive to the $B_{s}$ decay for $B r>1.2(0.65) \times 10^{-8}$ for the CDF (or combined CDF and D0) data, and this would cover a large part of the parameter space for $\tan \beta>30$. Non-universal models in the Higgs and third generation soft breaking masses offer the possibility of a new $Z$ boson s-channel annihilation in the early universe. Such possibilities can significantly increase the neutralino-proton detection cross section up the DAMA region of $10^{-6} \mathrm{pb}$. 


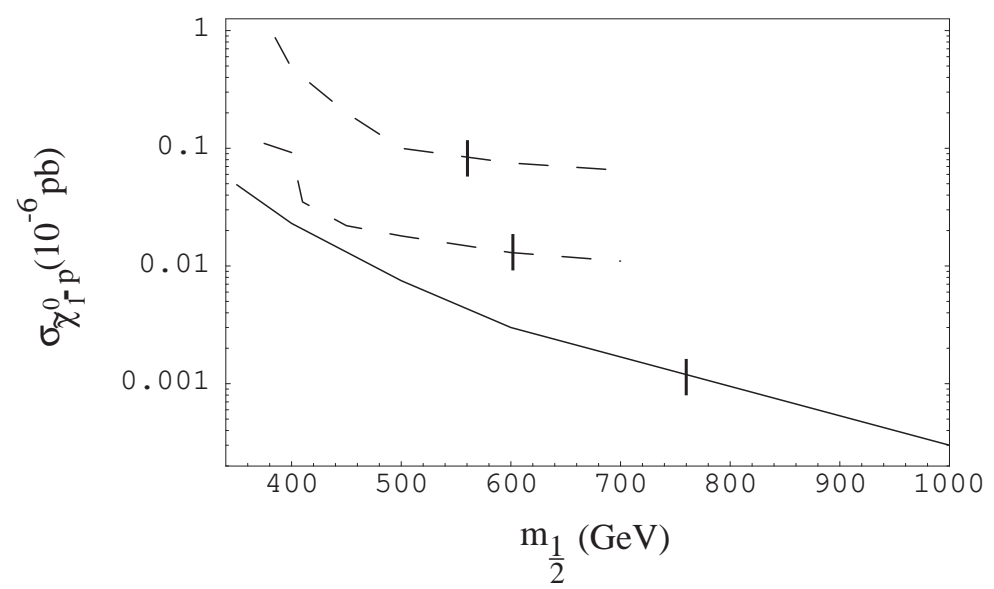

Figure 10: $\sigma_{\tilde{\chi}_{1}^{0}-p}$ as a function of $m_{1 / 2}\left(m_{\tilde{\chi}_{1}^{0}} \cong 0.4 m_{1 / 2}\right)$ for $\tan \beta=40, \mu>0, m_{h}>114$ $\mathrm{GeV}, A_{0}=m_{1 / 2}$ for $\delta_{2}=1$. The lower curve is for the $\tilde{\tau}_{1}-\tilde{\chi}_{1}^{0}$ co-annihilation channel, and the dashed band is for the $Z$ s-channel annihilation allowed by non-universal soft breaking. The curves terminate at low $m_{1 / 2}$ due to the $b \rightarrow s \gamma$ constraint. The vertical lines show the termination at high $m_{1 / 2}$ for $\delta a_{\mu}>11 \times 10^{-10}[24]$.

\section{Acknowledgement}

This work was supported in part by National Science Foundation Grant PHY-0101015.

\section{References}

[1] A.H. Chamseddine, R. Arnowitt, P. Nath, Phys. Rev. Lett. 49 (1982) 970.

[2] R. Barbieri, S. Ferrara, C.A. Savoy, Phys. Lett. B119 (1982) 343; L. Hall, J. Lykken, S. Weinberg, Phys. Rev. D27 (1983) 2359; P. Nath, R. Arnowitt, A.H. Chamseddine, Nucl. Phys. B227 (1983) 12.

[3] R. Rattazi, U. Sarid, Phys. Rev. D53 (1996) 1553.

[4] M. Carena, M. Olechowski, S. Pokorski, C. Wagner Nucl. Phys. B426 (1994) 269.

[5] G. Degrassi, P. Gambino, G. Giudice, JHEP 0012 (2000) 009.

[6] M. Carena, D. Garcia, U. Nierste, C. Wagner, Phys. Lett. B 499 (2001) 141.

[7] R. Arnowitt, B. Dutta, Y. Santoso, hep-ph/0010244; hep-ph/0101020; Nucl. Phys. B 606 (2001) 59. 
[8] J Ellis, T. Falk, G. Ganis, K. Olive, M. Srednicki, Phys. Lett. B 570 (2001) 236; J. Ellis, T. Falk, K. Olive, Phys. Lett. B 444 (367) 1998; J. Ellis, T. Falk, K. Olive, M. Srednicki, Astropart. Phys. 13 (2000) 181; Erratum-ibid.15, (2001) 413.

[9] M. Gomez, J. Vergados, hep-ph/0012020; M. Gomez, G. Lazarides, C. Pallis, Phys. Rev. D61 (2000) 123512; Phys. Lett. B487, (2000) 313; L. Roszkowski, R. Austri, T. Nihei, JHEP 0108, (2001) 024; A. Lahanas, D. Nanopoulos, V. Spanos, Phys. Lett. B 518 (2001) 94 and talk by V. Spanos at this conference.

[10] See e.g., M.B. Green, J.H. Schwarz, E. Witten, "Superstring Theory", vol.2, Sec.16.4 (Cambridge University Press, Cambridge, 1987).

[11] P. Igo-Kemenes, LEPC meeting, November 3, 2000 (http://lephiggs.web.cern. ch/LEPHIGGS/talks/index.html).

[12] M. Carena, M. Quiros and C. Wagner, Nucl. Phys. B461 (1996) 461;S. Heinemeyer, W. Hollik and G. Weiglein, Phys.Commun 124 (2000) 76.

[13] M. Alam etal Phys. Rev. Lett.74 (1995) 2885.

[14] M. Turner, astro-ph/0202007.

[15] J. Ellis, A. Ferstl, K. A. Olive, Phys. Lett. B 481 (2001) 304; Phys. Rev. D 63 (2001) 065016.

[16] V. Bednyakonv, hep-ph/0201046.

[17] H.N. Brown et.al., Muon (g-2) Collaboration, Phys. Rev. Lett.86, (2001) 2227.

[18] M. Knecht, A. Nyffeler, hep-ph/0111058; M. Knecht, A. Nyffeler, E. De Raphael, hepph/0111059; M. Hayakawa, T. Kinoshita, hep-ph/0112102; I. Blokland, A. Czernecki, K. Melnikov, hep-ph/0112117.

[19] CMD-2 Collaboration, Phys. Lett. B 5271 (2002) 161.

[20] N.M.A. Achasov etal., hep-ex/0201040; hep-ex/0202032.

[21] BES Collaboration, hep-ex/0105074.

[22] T.C. Yuan, R. Arnowitt, A.H. Chamseddine, P. Nath, Z. Phys. C 26 (1984) 407.

[23] D.A. Kosower, L.M. Krauss, N. Sakai, Phys. Lett. B 133 (1983) 305.

[24] R. Arnowitt, B. Dutta, B. Hu and Y. Santoso, Phys. Lett. B 505 (2001) 177.

[25] J.L. Lopez, D.V. Nanopoulos, X. Wang, Phys. Rev. D 49 (1994) 366. 
[26] U. Chattopadhyay and Pran Nath, Phys. Rev. D 53 (1996) 1648.

[27] K.S. Babu and C. Kolda, Phys. Rev. Lett. 84 (2000) 228; P.H. Chankowski and L. Slawianowska, Acta Phys. Polon. B 32 (2001) 1895; C.S. Huang and W. Liao, Phys. Rev. D 63 (2001) 11402; (E) D64 (2001) 059902; hep-ph/0201121; G. Isidori and A. Retico, JHEP 0111 (2001) 001.

[28] C. Bobeth, T. Ewerth, F. Kruger and J. Urban, Phys. Rev. D 64 (2001) 074014.

[29] A. Dedes, H.K. Dreiner and U. Nierste, Phys. Rev. Lett. 87 (2001) 251804.

[30] R. Arnowitt, B. Dutta, T. Kamon and M. Tanaka; hep-ph/0203069.

[31] P. Belli, R. Cerulli, N. Fornengo, S. Scopel, hep-ph/0203242 